\title{
A virtual clinic to improve long-term outcomes in chronic kidney disease
}

\author{
Authors: Patrick Harnett, ${ }^{\mathrm{A}}$ Matthew Jones, ${ }^{\mathrm{B}}$ Michael Almond, ${ }^{\mathrm{C}}$ Gowrie Ballasubramaniam ${ }^{\mathrm{D}}$ and Vinni Kunnath ${ }^{\mathrm{E}}$
}

\begin{abstract}
Chronic kidney disease (CKD) is common. A small proportion of patients with CKD progress to require interventions, which may include dialysis. Monitoring patients with CKD is supported by national guidelines. Monitoring systems to plan management of CKD vary in form. A novel monitoring system, the virtual CKD clinic (VC) was introduced at our hospital. The VC is a non-face-to-face results review of patients with CKD. We found that the VC was an effective monitoring system. None of the patients from the VC required emergency dialysis, suggesting robust surveillance. Survival was similar to patients with CKD discharged to primary care.
\end{abstract}

KEYWORDS: Virtual clinic, non face-to-face, chronic kidney disease, dialysis crash lander, computer decision support system

\section{Introduction}

Chronic kidney disease (CKD) is common in the UK with estimates of prevalence for all CKD (stage 1 to 5 ) of $14 \%$ in males and $13 \%$ in females. Late stage CKD (stages 4 and 5 ) is estimated at $6 \%$. The incidence of CKD increases with age and comorbidity. The most frequent causes of dialysis-dependant CKD in the UK are diabetes, hypertension, and vascular disease. ${ }^{2}$ A small proportion of patients with CKD will progress to require renal replacement therapy. ${ }^{3}$

Systems of surveillance for patients with CKD vary depending on local practice. Most of the surveillance occurs face-to-face in primary care or in hospital outpatients. The objectives of surveillance of CKD is to arrange timely preparation for renal replacement therapy and to avoid unplanned emergency dialysis, which is associated with worse survival, ${ }^{4}$ or to prepare for palliative non-dialysis care. Variation in incidence rates of unplanned dialysis is wide. ${ }^{5}$ A number of mechanisms have been

Authors: ${ }^{\mathrm{A}}$ consultant acute physician and nephrologist, Chelsea and Westminster Hospital, London, UK; ${ }^{\text {B }}$ data manager, Southend University Hospital NHS Foundation Trust, Southend-on-Sea, UK; ' Consultant physician, Royal Airforce Medical Reserve, UK; ${ }^{D}$ consultant physician and nephrologist, Southend University Hospital NHS Foundation Trust, Southend-on-Sea, UK; Erenal nurse specialist, Southend University Hospital NHS Foundation Trust, Southend-on-Sea, UK proposed in order to improve surveillance for advancing CKD and improve outcomes.

In the UK in 2006 the Royal college of Physicians (RCP) and the Royal college of General Practitioners (RCGP) published comprehensive guidance on the management of CKD. ${ }^{6}$ The RCP guides adopted the US 2002 National Kidney Federation Kidney Disease Outcomes Quality Initiative (NKF KDOQI) CKD classification system and management guidelines. ${ }^{7}$

The 2006 RCP/RCGP CKD guidelines proposed a collaborative approach between primary and secondary care for surveillance and management of CKD. The guidelines propose a 'virtual nephrologist' model of care, stating that

There are important options for computerised decision support including the virtual nephrologist model, which requires computerised transfer of information including laboratory measurements, between the GP surgery, the local laboratory and the nephrology service, and division of responsibility for acting on such results clearly identified in the care plan.

The guidelines were not specific on the definition of the virtual nephrologist. In our renal unit virtual CKD clinics (VCs) were proposed as a mechanism for improving monitoring of CKD. Our VC model was designed to utilise information technology (IT) and computer-based systems and therefore could be regarded as computerised decision support system (CDSS) for CKD monitoring. While strongly reliant on IT, the design of the VC had consultantled decisions and review at the core of the process.

The 2006 RCP guide has since been superseded by the National Institute for Health and Care Excellence (NICE) guidelines on CKD 2014. ${ }^{8}$

CDSSs have been defined as computerised software systems designed to assist clinicians with decision-making tasks. ${ }^{9}$ Uptake of novel systems and proof of concept can be very slow in health systems. Some sources state that the time between demonstrated benefit of an innovation and widespread introduction of the innovation to be 17 years. ${ }^{9}$ Further subtypes of CDSS have been described. Systems can be passive (users explicitly make a request for support), semi-active (watchdog systems are invoked automatically and present information when users request it) or active (triggered automatically, present information without it being requested and, in some cases, make decisions without the intervention of clinicians). The VC model developed in our unit is an active form of clinical decision support system (CDSS).

A number of forms of CDSS for CKD management have been described. Abdel-Kader et al conducted a trial of a passive alert 
based system of alerts for patients with CKD 3 and 4 in primary care. ${ }^{10}$ A non-significant increase in the rate of proteinuria testing was seen but failed to increase referrals or use of angiotensinconverting-enzyme (ACE) inhibition. No other outcomes were reported. They hypothesised that little improvement occurred because of the preceding change to eGFR testing which had increased surveillance and referral for CKD patients from primary care physicians. Other projects have demonstrated considerable benefits of surveillance coupled with education. Rayner et al improved vascular access at the start of dialysis and improved end-of-life care with a broad-ranging community-wide CKD management project. ${ }^{11}$

The benefit of CDSS in this context has not been demonstrated convincingly for clinical outcomes such as survival or proportion of patients having unplanned dialysis.

The published literature shows a proliferation in the number type and intention of computerised systems to aid decisions and chronic health care and kidney disease. ${ }^{12} \mathrm{~A}$ variety of information technology platforms have been utilised in the assistance of managing CKD. Education, drug adherence and reconciliation and lifestyle advice are frequent themes. The iNephro project examined the use of a smartphone app to encourage drug adherence in patients with CKD. ${ }^{13}$ A large number of downloads (over 11,000) were followed by rapid decline in use, less than $10 \%$ by 1 year.

The aim of our VC is to provide and ensure a robust monitoring system for patients with CKD using non-face-to-face computerbased decision support with the intention of improving recognition of patients with deteriorating CKD, initiation of appropriately timed interventions and reduction of late presentation.

\section{Methods}

The Southend Renal Unit provides adult renal services to a population of approximately 330,000 people in south east Essex, UK.

The Southend Virtual Renal Clinic (VC) was commenced in 2006 following the publication of the RCP/RCGP guidelines on the management of CKD.

Selection criteria for the VC:

$>$ non-progressing or stable CKD

> capacity to understand and adhere with the VC monitoring requirements

> absence of other reasons to mandate conventional follow-up (eg active comorbidities).

The RCP/RCGP CKD guidelines 2006 define progression as a reduction in eGFR of greater than $5 \mathrm{~mL} / \mathrm{min} /$ year. All patients transferred to the VC had the monitoring process described and explained face-to-face by a consultant physician in a conventional clinic setting. On the point of transfer to VC, return to face-toface clinic criteria were discussed with the patients. The prompt availability of return to conventional face-to-face review clinic was emphasised at the discussion of transfer to VC.

All patients discharged from the single clinic that were eligible were offered VC follow-up. Patients ineligible (eg eGFR decline over $5 \mathrm{~mL} / \mathrm{min} / 1.73 \mathrm{~m}^{2} /$ year or other reason) remained in conventional face-to-face follow-up.

On entry to the VC, patients were given blood (electrolytes and creatinine) and urine (urine protein:creatinine ratio) test request forms. Patients were instructed to have their tests 1 month prior to the date of the review.
The VC was developed with full support and knowledge of primary care. The availability of the VC was publicised in a series of lectures, discussions and visits primary care practices in the region. The VC was supported by the hospital trust with IT support, nurse practitioner assistance and dedicated consultant time.

As part of the VC 'package' primary care was offered a 'hotline' phone number to allow access to consultant advice for questions about patients with CKD. The consultant sessions also provided rapid access for patients requiring review when returning to faceto-face consultant clinic from the VC.

The VC was initiated according to national guidelines (RCP/RCGP CKD guidelines 2006) for local service development and service improvement. Ethical approval was not required as this service development conformed to national guidelines.

Patients were scheduled to have regular non-face-to-face test result reviews in the month after each test using the electronic pathology reporting system. Patients and their primary care doctors were informed of the outcome of the review by structured letter.

One of the following outcomes or actions was chosen based on the results.

> Repeat tests at standard frequency according to the CKD guidelines with subsequent non face-to-face review.

> Repeat test at shortened interval (if deterioration suspected) with subsequent non-face-to-face review.

$>$ Recall for face-to-face review in conventional clinic.

$>$ Reminder letters were sent if the patient had failed to have the required blood tests.

$>$ Discharge (if another significant life-limiting disease diagnosed).

$>$ Deceased / receiving palliative care.

The patient and primary care letter contains results (eGFR, Hb, urine protein:creatinine ratio) and includes blood and urine test forms for the next review. The review date was included in the letter.

Failure to undergo testing was followed by a structured reminder letter. Failure to undergo testing after three reminders was interpreted as a loss to follow-up. This was accompanied by a letter to the patient and to the primary care practice. Discussion with primary care physician was conducted if the patient was felt to be at risk (eg advanced stage CKD or known comorbidity).

The VC is based on human-led decision making and as such clinical opinion was also considered when movement between virtual and hospital care was undertaken. Some knowledge of the patients, their records and other elements such as records of unplanned attendances were available to inform the decisions. Utilisation of a renal database (Clinical Computing PROTON ${ }^{\circledR}$ ) and a graphical function on the pathology reporting system (Sunquest ICE $^{\circledR}$ ) were also considered.

Deterioration in tests was said to be present if:

> a deterioration of estimated glomerular filtration rate (eGFR) of greater than $5 \mathrm{~mL} / \mathrm{min} / 12$ months

> the arrival of patient at CKD stage 4 when further deterioration was thought to be likely

$>$ recurrence or development of proteinuria

$>$ progression of urine protein to creatinine ratio to levels over 100 $\mathrm{mg} / \mathrm{mmol}$

> communication from patient or primary care doctor regarding symptoms. 


\section{Comorbidities}

Common comorbidities (peripheral vascular disease, coronary disease, cerebrovascular disease, diabetes, chronic obstructive pulmonary disease [COPD], hypertension and malignancy) were recorded as present when documentary evidence was seen in the electronic record.

\section{Sample collection}

A robust system of community phlebotomy and pathology sample retrieval was in place in the region. All samples (community or hospital) were processed by the same laboratory at Southend Hospital and results were stored on a single pathology system (Sunquest integrated clinical environment, ICE) that provided data to both primary and secondary care. Retrieval of laboratory results was robust and reliable.

\section{Data collection}

Electronic patient records (patient administration system $\left[\mathrm{PAS}^{\circledR}\right]$, integrated clinical environment [ICE ${ }^{\circledR}$ ]) were used to collate clinical, comorbidity, demographic and laboratory data for all patients in the VC from July 2006 to March 2015.

\section{Outcomes}

The outcome measures were recorded included patient survival, requirement for renal replacement and unplanned emergency dialysis. Measurements of process included frequency of monitoring of relevant tests compared to guidelines.

Survival data regarding patients discharged to primary care from a conventional renal clinic in the same department under a different nephrologist were retrieved. Patients from this clinic with stable CKD were discharged to primary care with instructions for monitoring according to the RCP/RCGP guidelines. Survival rates in the VC were compared to survival rates in the VC using a chi-squared method to test the hypothesis that there was no difference in survival between groups.

Diagnoses, comorbidities and outcomes were retrieved from electronic records and HES data (Hospital Episode Statistics - NHS Digital).

\section{Results}

A total of 683 patients were reviewed in the VC between July 2008 and March 2015. Over this period, 5,024 non-face-to-face 'virtual' reviews were conducted.

Forty-five percent were female. The median age at first review was 78.5 years (range 17-90). Nine percent were under 40 years old; $43 \%$ between 40 and 75 years old and $48 \%$ over 75 . Table 1 shows the number of patients in each CKD stage at entry to the VC. Mean eGFR on entry to VC was $41.3 \mathrm{~mL} / \mathrm{min}$ (standard deviation $[S D]=25)$. At entry the average stage of CKD was stage 3 .

Seventy-six percent were over 60 years old. The mean duration of monitoring for a patient in the VC was 42.5 months (SD $=30$ months).

\section{Primary renal diagnosis of patients in the VC}

The majority of patients ( $72 \%$ ) were diagnosed as chronic renal disease of uncertain aetiology ( 494 patients in total).
Forty-six patients were diagnosed with renovascular disease (7\%). Thirty-eight had diabetic nephropathy (5\%) out of 111 with diabetes (16\%). Twenty-nine patients had polycystic kidney disease $(4 \%)$.

\section{Comorbidities of patients in the VC}

Hypertension was present in 318 patients ( $47 \%$ ). Ischaemic heart disease was present in 122 (18\%).

Peripheral vascular disease was present in 47 patients (7\%) with cerebrovascular disease in 59 (9\%). A diagnosis of malignancy was present in 68 patients (10\%). COPD had been diagnosed in 34 (5\%).

Atrial fibrillation was present in 41 patients and aortic stenosis or valve replacement in 15; dementia was diagnosed in 18; gout was present in 36 patients; congestive cardiac failure in 16 .

\section{Comorbidity burden}

The presence of the most frequent comorbidities was recorded; this consisted of hypertension, ischaemic heart disease, peripheral vascular disease, malignancy, stroke, diabetes and COPD. The burden of comorbidity by patient numbers is shown in Table 2 . Only 248 patients had no recorded comorbidities. Most patients had between one and three comorbidities.

\section{Cardiovascular comorbidity}

388 of 683 (57\%) patients had one or more cardiovascular comorbidities while 196 patients had two or more (28\%). 60 patients had three or more cardiovascular comorbidities ( $9 \%$ ).

\section{Monitoring}

The annual frequency of testing for CKD patients was recorded and compared to the recommended test frequency from the RCP and RCGP guidelines (see Table 3). Overall, patients received testing more frequently than the recommended guidelines.

\section{Outcomes}

Survival

By March 2015, 218 patients had died. The average age at death was 81 -years-old $(S D=9.4$ years). Mean duration of VC follow-up was 28.4 months.

\section{Survival in patients with standard care compared to VC (see Table 4)}

Survival data on patients reviewed in the VC was compared to standard care (patients discharged from a general renal clinic in the same unit that were followed up in general practice). Of patients in the VC, $31.9 \%$ had died by the end of the data collection period. When compared to the proportion of patients surviving after discharge to general practice according to guidelines, no significant difference in survival rates was apparent at individual stages. A survival benefit for the VC compared to standard care was apparent over all stages of CKD combined.

Patients from the VC going on to require renal replacement therapy (see Table 5)

Twenty-seven patients required renal replacement therapy; 26 had dialysis and one had a preemptive renal transplant. Nineteen 
patients had haemodialysis and seven had peritoneal dialysis.

Twenty patients out of 26 started dialysis with definitive dialysis access; six had semi-permanent dialysis lines. One patient required urgent placement of the line. No temporary dialysis catheters were used and no out-of-hours / emergency dialysis was required.

\section{Discussion}

This article describes the patient characteristics and outcomes of the VC, a computer-assisted non-face-to-face CKD monitoring system.

The spectrum of age, severity and comorbidity in our VC is similar to that found in published data from other developed countries. ${ }^{14}$

No significant difference in survival was demonstrated for the different stages of CKD. Overall survival was better in the VC than standard care. The difference reaches statistical significance, but clinical significance is less clear. This study retrieved the data in retrospect. The data is observational and not a randomised comparison and as such the inference of a survival benefit has only limited strength.

Twenty-seven patients required renal replacement therapy. All of the patients emerging from the VC and going on to need renal replacement therapy did so in a planned and controlled manner. Unplanned start for dialysis is said to occur in patients with CKD that are started on dialysis as an emergency. It is also colloquially referred to as 'crash landing'. There were no late presentations 'dialysis crash landers' from the VC.

A high proportion of patients had good quality of access (fistula or peritoneal dialysis [PD] catheter) when starting dialysis from VC. The rates for fistula formation and PD catheter placement in patients from the VC were higher than the in the region and the national average, see Table 6 (data from renal registry multisite access audit $2014^{2}$ ).

The 2006 KDOQI guidelines suggest that 'patients should have a functional permanent access at the initiation of dialysis therapy'? No consensus definition of unplanned dialysis exists.

The renal registry record access type of in the UK multisite dialysis access audit $2015 .{ }^{5}$ Use of non-tunnelled lines in patients with CKD requiring dialysis is a surrogate marker for unplanned dialysis. The rates of non-tunnelled line use are highly variable across the UK. Late referral to renal services is considered as a factor contributing to the proportion of patients having suboptimal access. Patients receiving dialysis via non-tunnelled lines despite being known to renal services is also a marker of unplanned dialysis.

Table 1. Number of patients by chronic kidney disease (CKD) stage at entry to the virtual clinic

\section{(VC)}

\section{CKD stage at entry to VC}

CKD stage 1

CKD stage 2

CKD stage 3

CKD stage 4

CKD stage 5
Number of patients

40

72

300

237

25
Table 2. Burden of comorbidity by patient numbers

\begin{tabular}{ll} 
Comorbidities per patient & Number of patients \\
0 & 248 \\
1 & 200 \\
2 & 143 \\
3 & 156 \\
4 & 18 \\
5 & 5 \\
\hline
\end{tabular}

Other definitions or markers of unplanned dialysis have been suggested. Brown et al defined unplanned dialysis as starting chronic dialysis as an inpatient. ${ }^{15}$ In their retrospective analysis unplanned dialysis was associated with diabetes, heart failure and high body mass index. Absence of predialysis education was also found to be associated with unplanned start in addition to medical comorbidity.

Buck et al defined classified unplanned start as 'known acute patients' that had been encountered by renal services for over 4 months prior to starting dialysis as an emergency. ${ }^{16}$ Unplanned dialysis is strongly associated with poor survival following initiation of renal replacement therapy (RRT). ${ }^{17}$ Kessler et al found a strong relationship for length of time between referral and initiation of RRT and short-term survival after RRT. ${ }^{18}$ In $2015,13.9 \%$ of patients known to UK renal services for more than 90 days started haemodialysis in this cohort did so with a non-tunnelled line.

\section{Initial access for patients starting dialysis}

Our of 19 patients starting haemodialysis from the VC, 13 started with a functioning arteriovenous (AV) fistula (68\%).

Renal Association guidelines state that $65 \%$ of patients commencing haemodialysis should start with an AV fistula. ${ }^{19}$ There is wide regional variation with lower than $15 \%$ fistula rate seen in some centres. Only five centres in the UK achieved the target of $65 \%$ of patients starting dialysis with an AV fistula. The national audit report accepts that the data do not explain the reasons for the wide variation in attainment of the target.

Referral time has a strong influence on the type of starting access for both PD and haemodialysis. ${ }^{20}$ Buck et al also found that a strong predictor for starting with definitive access was attending predialysis clinics. ${ }^{16}$ Patients who did not attend a predialysis clinic had a $90 \%$ increase in the odds of having an urgent known acute dialysis initiation.

Patients with polycystic kidney disease (PKD) have a more predictable course of deterioration in renal function and had the highest chance of starting haemodialysis with a fistula (66.1\%). PKD patients are also more likely to be known to renal services.

Patients with PKD represented $4.5 \%$ of the total number of patients starting dialysis and $3.8 \%$ of those starting on haemodialysis in the UK. ${ }^{2}$ In the VC population, 29 of 693 patients $(4.2 \%)$ had PKD. PKD is not over-represented in the VC; therefore, it is not an explanation for the low incidence of crash landing.

Non-linear progression of renal disease and abrupt changes due to intercurrent illness contribute to the challenge in preparing patients at an appropriate time for renal replacement. The consequences for patients of late presentation are severe and include a reduced survival on dialysis. Therefore, robust 
Table 3. Annual frequency of monitoring in the virtual clinic (VC) compared to Royal College of Physicians / Royal College of General Practitioners guidelines and National Institute of Health and Care Excellence guidelines 2014

\begin{tabular}{lllllll} 
& & & \multicolumn{4}{c}{ NICE 2014 } \\
\cline { 3 - 5 } eGFR & CKD Stage & RCP/RCGP 2006 & ACR $<3$ & ACR 3-30 & ACR $>30$ & Frequency of VC tests \\
$>90$ & 1 & 1 & $<1$ & 1 & $>1$ & 2.27 \\
$60-89$ & 1 & 1 & $<1$ & 1 & $>1$ & 2.77 \\
$45-59$ & 2 & 1 & 1 & 1 & 2 & 4.35 \\
$30-45$ & 3 & 2 & $<2$ & 2 & 3 & 6.38 \\
$15-30$ & 4 & 4 & 2 & 2 & $>4$ & 10.8 \\
$<15$ & 5 & 8.6 & 4 & $>4$ & 2 & \\
\hline
\end{tabular}

Observed VC monitoring frequency compared to frequency of monitoring of chronic kidney disease according to stage (NICE guidelines 2008 adopt the same advice as RCP/RCGP guidelines 2006. NICE 2014 based on KDIGO 2012 guidelines changed the recommended frequency of testing and included level of albuminuria as a factor. $A C R=$ albumin to creatinine ratio $\mathrm{mg} / \mathrm{\mu mol}$

surveillance systems are important in an attempt to produce a consistent approach to the reduction of poor outcomes in this small but important group. The VC may represent a scalable and transferrable technique that could contribute to reducing the occurrence of unplanned dialysis.

Other large-scale and important studies continue to investigate the benefit of virtual CKD monitoring. The ASSIST-CKD project is a UK-wide study sponsored by Kidney Research UK to 'spread eGFR graph surveillance for the early identification, support and treatment of people with progressive chronic kidney disease. ${ }^{21}$ The project involves 20 renal centres in the UK with the objective of identifying those patients at greatest risk of progression in order to deliver more timely treatment. This large and well-designed project will further clarify the role of surveillance and improve the ability of clinicians to add value to the CKD patient journey.

Just over $4 \%$ of the patients in the VC went on to receive RRT demonstrating that RRT requirement is a rare event in this group. The VC is intended as a surveillance technique and as such could be said to be effective. A technique that further refines and improves the predictive process is clearly felt to be desirable by the ASSIST CKD group and the wider nephrology community.

Almost one-third of patients died during the period of observation (32\%). The Rayner study demonstrated a great benefit in that a large proportion of patients receiving palliative care died out of hospital. ${ }^{11}$ The data from the VC did not include place of death or involvement of palliative care services. Modifications to the VC process could include this element of care and could add more value to non-face-to-face surveillance.

The VC is less resource intensive than face-to-face clinics. It circumvents the need for patients to travel to the hospital for a face-to-face review of tests, permitting less disruption to work schedules and less inconvenience for patients, particularly those with other comorbidities. It may represent an additional tool to extend contact with renal patients in a cost-efficient way, which encourages participation and adherence. High levels of acceptance and participation may be attributable to the flexibility of timing of tests, the convenience of the local infrastructure and the reminder system that is integral to the process.

The observed frequency of blood tests in the VC exceeds that of the frequency recommended in the guidelines. The 2006 Joint RCP and RCGP Guideline Development Committee (GDC) specified a frequency of testing dependent on stage of CKD.

This was based on consensus and accompanied by a statement that frequency of testing should be adjusted according to clinical judgment. Recommendation 9 from the guidelines states

exact frequency should depend on the clinical situation. The frequency of testing may be reduced if the eGFR levels remain very stable but will need to be increased if there is rapid progression.

Table 4. Survival of patients in in the virtual clinic (VC) compared to patients with chronic kidney disease (CKD) discharged to primary care according from the conventional renal clinic (RC) to CKD stage

\begin{tabular}{|c|c|c|c|c|c|c|c|c|c|}
\hline CKD stage & VC alive & Total & RC alive & Total & VC alive (\%) & RC alive (\%) & df, number & $\chi^{2}$ value & $p$ value \\
\hline 1 & 36 & 41 & 4 & 4 & 87.80 & 100 & $(1, n=45)$ & 0.55 & 0.46 \\
\hline 2 & 76 & 86 & 5 & 7 & 88.37 & 71.42 & $(1, n=93)$ & 1.65 & 0.2 \\
\hline 3 & 195 & 262 & 34 & 46 & 74.42 & 73.91 & $(1, n=308)$ & 0.01 & 0.94 \\
\hline 4 & 125 & 209 & 55 & 89 & 59.80 & 61.79 & $(1, n=298)$ & 0.1 & 0.74 \\
\hline 5 & 33 & 85 & 33 & 71 & 38.82 & 46.47 & $(1, n=156)$ & 0.92 & 0.33 \\
\hline Total & 465 & 683 & 131 & 217 & 68.08 & 60.36 & $(1, n=900)$ & 4.38 & 0.036 \\
\hline
\end{tabular}




\begin{tabular}{ll} 
Table 5. Number of patients starting renal \\
replacement therapy (RRT) from the virtual clinic \\
according to starting modality \\
\hline Starting modality & Number of patients \\
Pre-emptive transplant & 1 \\
Peritoneal dialysis & 7 \\
Haemodialysis & 19 \\
Primary fistula & 13 \\
Tunnelled line & 6 \\
Temporary line & 0 \\
Urgent dialysis out of hours & 0
\end{tabular}

NICE CKD guidelines 2008 clinical guideline 73 adopted the same recommendations for frequency of testing as the RCP 2006 guidelines.

Revision of the NICE CKD guidelines in 2014 adjusted the advice to increase frequency of testing where depending on the urinary albumin excretion levels. The guidelines were published in July 2014. The data set considered in this study concluded in March 2015. Changes in monitoring frequency recommended in these guidelines did not affect the patients in this data set.

The 2014 guidelines discuss at appropriate length the frequency of testing and the number of factors associated with progression and outcome. The non-linearity of progression of CKD is emphasised, with advice regarding the significance of increases and decreases to eGFR of $25 \%$ associated with increase mortality risk. The guidance also states the importance that the clinician and patient recognise that changes in the plus or minus $25 \%$ range can be explained by error and natural variability. The frequency of testing is derived from the 2012 KDIGO guidelines, which in turn are based on expert opinion. They go on to state

There are many who would like more definitive guidance on frequency of measurement according to specific categories of risk. However, this is not possible at the current time given the lack of evidence to guide such statements and the extreme number of individual circumstances that would mitigate any proposed protocol. $^{22}$
Adherence to requests for blood sampling was very high. Patient engagement in the VC is high. The regional phlebotomy infrastructure allows for convenient blood sampling and may facilitate participation and adherence to VC surveillance.

\section{Virtual clinics in other centres}

Early models of virtual renal clinics were acknowledged and promoted in a report by a joint working party by the RCP and the NHS Alliance in 2004. Clinicians, services and commissioning in chronic disease management in the NHS: The need for coordinated management programmes ${ }^{23}$ offers a definition of chronic disease management programmes based on the Wagner ${ }^{24}$ model. The model describes a pathway to improved chronic disease management by the development of better self-management, clinical information systems, delivery system redesign, decision support, healthcare reorganisation and improved community resources. It also describes the implementation and delivery of chronic disease management programmes giving six examples of programmes from the UK.

The Southampton Integrated Monitoring of Nephrology $(\mathrm{SIMON})^{25}$ programme evaluated a CDSS that facilitated monitoring of patients with CKD. The programme involved shared care between general practice and hospital-based clinicians. Following an initial assessment visit, a nephrologist sees and advises on blood test results and blood pressure measurements without seeing the patient face-to-face. The SIMON scheme enables remote monitoring by nephrologists of patients with CKD, with computer-generated reminder letters to prompt regular follow-up. A retrospective case control technique was used. In addition to monitoring, some interventions to improve blood pressure by remote/home measurement showed an improvement in the intervention group. The system proved feasible with high levels of patient participation and adherence. Importantly it demonstrated a system that shared care was of benefit and feasible. There is reference in the RCP guidelines to the SIMON system, which is described as a development of virtual services.

Data on survival in patients with CKD that had not been referred to secondary care was published by Stevens et al. ${ }^{14}$ The study included patients with a creatinine level of $>180 \mu \mathrm{mol} / \mathrm{L}$ for men and $>135 \mu \mathrm{mol} / \mathrm{L}$ for women. This equates to an estimated GFR of $35 \mathrm{~mL} / \mathrm{min}$ by modified Modification of Diet in Renal Disease calculation. The patient population were from east Kent, UK and

Table 6. Rates of tunnelled lines, fistulas and pre-emptive transplants in patients requiring renal replacement therapy (RRT) from the virtual clinic compared to regional and national averages and UK best and worst performances (UK renal registry data ${ }^{5}$ )

$\begin{array}{llllll} & \text { VC (2006-15) (\%) } & \text { Region 2015 (\%) } & \text { UK average 2015 (\%) } & \text { UK best 2015 (\%) } & \text { UK worst 2015 (\%) } \\ \text { Non-tunnelled line } & 0 & 23.8 & 13.9 & 0 & 32.7 \\ \text { Tunnelled line } & 22.2 & 19 & 26.2 & 61.9 & 14 \\ \text { Fistula/graft } & 48 & 33.3 & 36.6 & 55.6 & 0 \\ \text { Peritoneal dialysis } & 37 & 23.8 & 23.4 & & \\ \text { Pre-emptive transplant } & 3.7 & 5 & 7.5 & & \\ \text { Total (N) } & 27 & 21 & 4050 & & \end{array}$

Data for patients known for more than 90 days 
were predominantly white (2001 census data showing that less than $1.3 \%$ coming from an ethnic minority).

The average age was 83 -years-old and during a mean average of 31.3 months of follow-up nearly $40 \%$ of the study population had died.

Drey et al reported a retrospective cohort study of 1,076 patients with CKD in the Hampshire area of the UK in $2003 .{ }^{26}$ The survival in all groups with CKD (creatinine of $1.7 \mathrm{mg} / \mathrm{dL}$ or $150 \mu \mathrm{mol} / \mathrm{L}$ ) was found to be $31 \%$ after a mean average follow up of 5.5 years. Thirty-nine patients required renal replacement therapy.

Ennis et al reported the effect of appending alerts to laboratory reports to physicians in a case control study. ${ }^{27}$ The endpoints measured were the frequency of blood test relating to CKD and their adherence to recommended test frequencies in guidelines (KDOQI/KDIGO) The tests include eGFR, parathyroid hormone, phosphate and other regularly tested analytes. The study covered large numbers of patients and practices; 12,353 subjects and 42,996 controls. Decision system reports did increase adherence to guidelines and was considered beneficial. The study was of short duration ( 0.73 years mean follow-up) and no clinical hard endpoints were considered.

No penalties were recorded for over-testing. No measurement of effect of false alarm or inappropriate activity generated by reports were considered.

The authors conclude that there were benefits of the simplicity of integration and scalability of their CDSS were its strengths but also emphasised that guideline authors may need to take these developments into consideration.

Significant effects have been demonstrated in previous studies. Rayner et al have demonstrated an improvement the treatment pathway going to palliative care and in local rates of starting dialysis on fistula or peritoneal dialysis associated with surveillance. ${ }^{11}$

National scale studies are also currently underway to explore the benefits of electronic surveilance. ${ }^{21}$

Unlike this study, no virtual CKD systems have demonstrated measurable benefit on the clinical endpoint of access quality when compared to local practice.

\section{Conclusions}

The virtual CKD clinic described here is a robust system for followup of patients with CKD. It is associated with good adherence from patients.

The low level of unplanned dialysis appears to be its main advantage.

As a pilot study it has the benefit of a long-term follow-up (7 years). It is a system that relies on standard infrastructure and could be reproduced and 'up-scaled' in locations where rates of unplanned dialysis exceed the expected or average national level. There appears to be no survival disadvantage when compared to conventional guideline-based primary care. The success may also be due to the incorporation of a human element in the decisionmaking pathway and the capacity to promptly recall patients to face-to-face hospital care.

\section{Acknowledgements}

The authors would like to thank Dr David Carmichael for his help and support.

\section{References}

1 Roderick P, Roth M, Mindell J. Prevalence of chronic kidney disease in England: Findings from the 2009 Health Survey for England. J Epidemiol Community Health 2011;65(Suppl 2):A12

2 Gilg J, Rao A, Fogarty D. UK renal registry 17th annual report: Chapter 1 UK renal replacement therapy incidence in 2012: National and centre-specific analyses. Nephron 2014;125: $1-27$.

3 Go A, Chertow G, Fan D, McCulloch C, Hsu C. Chronic kidney disease and the risks of death, cardiovascular events, and hospitalization. N Engl J Med 2004;351:1296-305.

4 Mendelssohn DC, Malmberg C, Hamandi B. An integrated review of 'unplanned' dialysis initiation: reframing the terminology to 'suboptimal' initiation. BMC Nephrol 2009;10:22.

5 Rao A, Evans R, Wilkie M, Fluck R, Kumwenda M. UK Renal Registry 18th Annual Report: Chapter 112014 Multisite Dialysis Access Audit in England, Northern Ireland and Wales and 2013 PD One Year Follow-up: National and Centre-specific Analyses. Nephron 2016;132:253-77

6 Burden R, Tomson C. Guideline Development Committee, Joint Specialty Committee on Renal Disease of the Royal College of Physicians of London and the Renal Association. Identification, management and referral of adults with chronic kidney disease: concise guidelines. Clin Med 2005;5:635-42.

7 National Kidney Foundation. Clinical Practice guidelines for chronic kidney disease: evaluation classification and stratification. National Kidney Foundation, Inc, 2002.

8 National Institute for Health and Care Excellence. Chronic kidney disease in adults: assessment and management [CG182]. NICE, 2014.

9 O'Sullivan D, Fraccaro A, Carson E, Weller P. Decision time for clinical decision support systems. Clin Med 2014;14:338-41.

10 Abdel-Kader K, Fischer GS, Lie ] et al. Automated clinical reminders for primary care providers in the care of CKD: a small clusterrandomized controlled trial. Am J Kidney Dis 2011;58:894-902.

11 Rayner H, Baharani J, Dasgupta I et al. Does community-wide chronic kidney disease management improve patient outcomes? Nephrol Dial Transplant 2014;29:644-9.

12 Diamantidis CJ, Becker S. Health information technology (IT) to improve the care of patients with chronic kidney disease (CKD). BMC Nephrol 2014;15:7.

13 Becker S, Kribben A, Meister S et al. User profiles of a smartphone application to support drug adherence - experiences from the iNephro project. PLoS One 2013;8:6-11.

14 Stevens PE, O'Donoghue DJ, de Lusignan S et al. Chronic kidney disease management in the United Kingdom: NEOERICA project results. Kidney Int 2007;72:92-9.

15 Brown PA, Akbari A, Molnar AO et al. Factors associated with unplanned dialysis starts in patients followed by nephrologists: a retropective cohort study. PLoS One 2015;10:1-10.

16 Buck J, Baker R, Cannaby AM et al. Why do patients known to renal services still undergo urgent dialysis initiation? A cross-sectional survey. Nephrol Dial Transplant 2007;22:3240-5.

17 Lorenzo V, Martn M, Rufino M et al. Predialysis nephrologic care and a functioning arteriovenous fistula at entry are associated with better survival in incident hemodialysis patients: an observational cohort study. Am J Kidney Dis 2004;43:999-1007.

18 Kessler M, Frimat L, Panescu V, Briançon S. Impact of nephrology referral on early and midterm outcomes in ESRD: EPidémiologie de I'Insuffisance REnale chronique terminale en Lorraine (EPIREL): results of a 2-year, prospective, community-based study. Am J Kidney Dis 2003;42:474-85.

19 Fluck R, Kumwenda M. Association Clinical Practice Guideline on vascular access for haemodialysis. Nephron Clin Pract 2011;118 (Suppl 1):c225-40. 
20 Roderick P, Jones C, Tomson C, Mason J. Late referral for dialysis: Improving the management of chronic renal disease. QJM 2002;95:363-70.

21 Gallagher H, Methven S, Casula A et al. A programme to spread eGFR graph surveillance for the early identification, support and treatment of people with progressive chronic kidney disease (ASSIST-CKD): protocol for the stepped wedge implementation and evaluation of an intervention to reduce late presentation for renal replacement therapy. BMC Nephrol 2017;18:1-10

22 Kidney Disease: Improving Global Outcomes (KDIGO) CKD Work Group. KDIGO 2012 Clinical Practice Guideline for the Evaluation and Management of Chronic Kidney Disease. Kidney Int Suppl 2013;3:4

23 Royal College of Physicians of London, the Royal College of Genera Practitioners and the NHS Alliance. Clinicians, services and commissioning in chronic disease management in the NHS. The need for coordinated management programmes. London: RCP, 2004.

24 Bodenheimer T, Eh W, Grumbach K. Improving primary care for patients with chronic illness. JAMA 2002;288:1775-9.
25 Jones C, Roderick P, Harris S, Rogerson M. An evaluation of a shared primary and secondary care nephrology service for managing patients with moderate to advanced CKD. Am J Kidney Dis 2006;47:103-14.

26 Drey N, Roderick P, Mullee M, Rogerson M. A population-based study of the incidence and outcomes of diagnosed chronic kidney disease. Am J Kidney Dis 2003:42:677-84.

27 Ennis J, Gillen D, Rubenstein A et al. Clinical decision support improves physician guideline adherence for laboratory monitoring of chronic kidney disease: a matched cohort study. BMC Nephrol 2015;16:163.

Address for correspondence: Dr Patrick Harnett, Acute Medicine, Chelsea and Westminster Hospital, Fulham Road, London SW10 9NH, UK.

Email: harnett101@gmail.com

\section{NGC

\section{New to systematic reviewing, or need to improve your critical appraisal skills?}

Our intensive 1-day courses will introduce you to the key principles of critical appraisal and systematic reviewing, and equip you with the basic skills to put your knowledge into practice.

> Introduction to critical appraisal

> Systematic reviews and meta-analysis in action

Discount available if both courses are booked together.

'Informative and practical' Dr Rajeswari Ramaraj
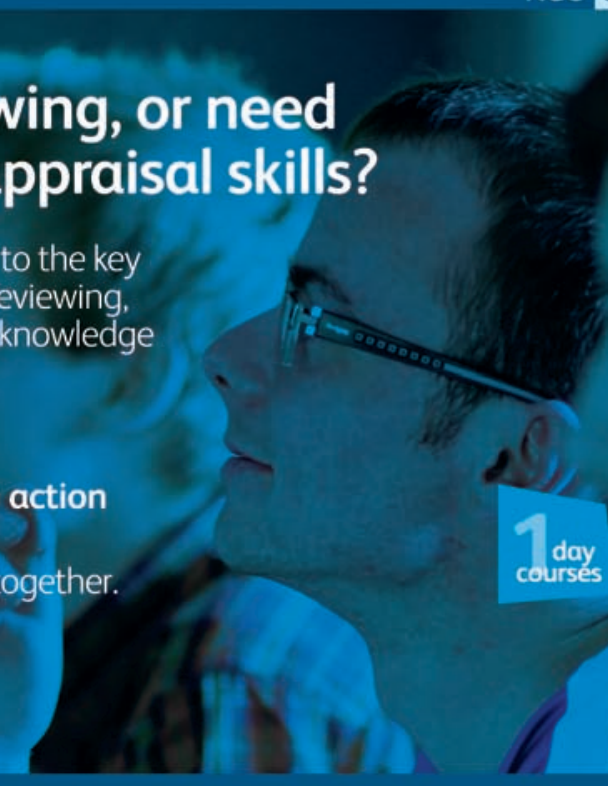

For course dates and more information visit: www.ngc.ac.uk/training-courses 